\title{
RINGS OF CONTINUOUS FUNCTIONS WITH VALUES IN A TOPOLOGICAL FIELD
}

\author{
BY \\ GEORGE BACHMAN, EDWARD BECKENSTEIN, LAWRENCE NARICI \\ AND SETH WARNER
}

\begin{abstract}
Let $F$ be a complete topological field. We undertake a study of the ring $C(X, F)$ of all continuous $F$-valued functions on a topological space $X$ whose topology is determined by $C(X, F)$, in that it is the weakest making each function in $C(X, F)$ continuous, and of the ring $C^{*}(X, F)$ of all continuous $F$-valued functions with relatively compact range, where the topology of $X$ is similarly determined by $C^{*}(X, F)$. The theory of uniform structures permits a rapid construction of the appropriate generalizations of the Hewitt realcompactification of $X$ in the former case and of the Stone-Čech compactification of $X$ in the latter. Most attention is given to the case where $F$ and $X$ are ultraregular; in this case we determine conditions on $F$ that permit a development parallel to the classical theory where $F$ is the real number field. One example of such conditions is that the cardinality of $F$ be nonmeasurable and that the topology of $F$ be given by an ultrametric or a valuation. Measure-theoretic interpretations are given, and a nonarchimedean analogue of Nachbin and Shirota's theorem concerning the bornologicity of $C(X)$ is obtained.
\end{abstract}

Our goal is to develop a theory parallel to that of realcompact spaces and realcompactifications by replacing the topological field $\mathbf{R}$ of real numbers with a complete topological field $F$, with emphasis on the case where $F$ is ultraregular. (An ultraregular space is a Hausdorff space for which the clopen sets form a basis, a clopen set being one that is both open and closed. An ultranormal space is a Hausdorff space each closed subset of which has a fundamental system of neighborhoods consisting of clopen sets. There exist ultraregular spaces that are not ultranormal [25] , [26].) Our emphasis on ultraregular fields is prompted by the fact that every field whose topology is given by a valuation or an ultrametric is ultraregular (and, in fact, is ultranormal).

If $X$ and $Y$ are topological spaces, $C(X, Y)$ denotes the set of all continuous functions from $X$ into $Y$, and $C^{*}(X, Y)$ denotes the set of all continuous functions $f$ from $X$ into $Y$ such that $\overline{f(X)}$ is compact. If $F$ is a topological field, $C(X, F)$ and $C^{*}(X, F)$ are both $F$-algebras; if, moreover, $F$ is locally compact,

Received by the editors July 24, 1973.

AMS (MOS) subject classifications (1970). Primary 54D35, 54D60, 54E15.

Key words and phrases. Topological field, realcompactification, ultraregular, ultranormal. 
$C^{*}(X, F)$ is the $F$-algebra of all bounded, continuous, $F$-valued functions on $X$. In $\S 1$ we generalize the Stone-Čech compactification and obtain ultraregular analogues of the Gelfand-Kolmogoroff theorem and the characterization of the Stone-Čech compactification $\beta X$ of $X$ as the suitably topologized space of characters of $C^{*}(X, \mathbf{R})$.

In $\S 2$ we develop a theory of $F$-repletions which generalizes that of realcompactifications by replacing $\mathbf{R}$ with a separated, complete uniform space $F$. The theory is developed by means of uniform spaces, but it is essentially equivalent to the theory of $E$-compactifications developed by Mrówka and others. The basic theory flows easily from this development which, in addition, enables us to give new proofs of known facts about $\mathrm{N}$-replete (i.e., $\mathrm{N}$-compact) spaces and new theorems about $F$-repletions (i.e., $F$-compactifications) for a large class of spaces $F$, including $\mathbf{N}$, and hence accounts for our choice. Moreover, this development seems particularly natural in view of the fact that the statement of the deepest and most important theorem about realcompactness, Shirota's theorem, involves uniform structures. (Following Bourbaki and others, we use " $F$-replete" and " $F$-repletion" rather than " $F$-compact" and " $F$-compactification" (although we use "realcompact" and "realcompactification") because the theory is developed here by means of uniform spaces, because the spaces encountered are quite remote from compact spaces, and because one cannot omit " $E$ " from " $E$-compact" or " $E$-compactification" without risking grave misunderstanding.)

Our central results are given in $\S 3$, where we specialize to the case where $F$ is a complete ultraregular field, especially one whose topology is given by an ultrametric or a valuation. In $\S 4$ we give a measure-theoretic interpretation to certain constructions, and in $\$ 5$ we present a nonarchimedean analogue of the theorem of Nachbin [20] and Shirota [28] characterizing those spaces $C(X, \mathbf{R})$ that are bornological for the topology of compact convergence.

1. The Banaschewski compactification. Let $X$ and $F$ be Hausdorff spaces. We denote by $C^{*}(X, F)$ the weakest uniform structure on $X$ making each $f \in$ $C^{*}(X, F)$ uniformly continuous into the compact space $\overline{f(X)}$, equipped with its unique compatible uniform structure. We shall say that $X$ is F-completely regular if $C^{*}(X, F)$ is compatible with the topology of $X$. By [5, Proposition 8, p. 203], $X$ is $F$-completely regular if and only if $X$ is homeomorphic to a relatively compact subset of the cartesian product $F^{B}$ for some set $B$. By [5, Proposition 3, p. 230], $C^{*}(X, F)$ is a precompact uniform structure. Thus if $X$ is $F$-completely regular, the completion $\beta_{F} X$ of $X$ for $C^{*}(X, F)$ is a compactification of $X$, and each $f \in$ $C^{*}(X, F)$ has a continuous extension to $\beta_{F} X$. It is easy to see that, if $X$ and $Y$ are $F$-completely regular spaces, each $f \in C(X, Y)$ is uniformly continuous for 
$C^{*}(X, F)$ and $C^{*}(Y, F)$ and hence has a continuous extension $\beta_{F} f: \beta_{F} X \rightarrow \beta_{F} Y$. In particular, every continuous function from $X$ into a compact, $F$-completely regular space has a continuous extension to $\beta_{F} X$.

The R-completely regular spaces are, of course, precisely the completely regular spaces, and, by definition, $\beta_{\mathrm{R}} X$ is the Stone-Čech compactification $\beta X$ of $X$ for any completely regular space $X$. If $F$ is ultraregular and contains at least two points, it is easy to see that $X$ is $F$-completely regular if and only if $X$ is ultraregular. In this case, $C^{*}(X, F)$, and hence also $\beta_{F} X$, may be described intrinsically, as follows: For each cover $V$ of $X$, we denote by $W(V)$ the subset $U\{V \times V: V \in V\}$ of $X \times X$. A partition $V$ of $X$ (that is, a cover of $X$ by mutually disjoint subsets) is open if each of its members is open (and hence closed). It is easy to verify that $\{W(V): V$ is a finite open cover of $X\}$ is a fundamental system of entourages for a uniform structure $B_{0}(X)$ on $X$; as $X$ is ultraregular, $B_{0}(X)$ is compatible with the topology of $X$. Moreover, each finite open cover $V$ consists of $W(V)$-small sets, so $B_{0}(X)$ is precompact, and therefore the completion $\beta_{0} X$ of $X$ for $B_{0}(X)$ is a compactification of $X$, which we shall call the Banaschewski compactification since it was first studied in [1]. One may readily show that if $X$ is ultranormal, then $C^{*}(X, \mathbf{R})=B_{0}(X)$ and hence $\beta_{0} X=\beta X$, by using the fact that an ultranormal space has zero Lebesgue covering dimension (i.e., every finite open cover is refined by a finite open partition) [21, 8-3]. More generally, if $F$ is ultraregular and has at least two points, one may easily show that $C^{*}(X, F)=B_{0}(X)$ and hence $\beta_{F} X=$ $\beta_{0} X$. If $Y$ is also ultraregular, it is natural in this case to denote the continuous extension of $f \in C(X, Y)$ to a function from $\beta_{0} X=\beta_{F} X$ into $\beta_{0} Y=\beta_{F} Y$ by $\beta_{0} f$ rather than $\beta_{F} f$.

If $C$ is a clopen subset of ultraregular $X$, its characteristic function is continuous from $X$ into $\{0,1\}$ and hence has a continuous extension to $\beta_{0} X$; it follows readily that the closure $\bar{C}$ of $C$ in $\beta_{\mathrm{o}} X$ is clopen [23, Lemma 1.7.1]. Consequently, if $V$ is a finite open partition of $X$, the set $\bar{V}$ of closures in $\beta_{0} X$ of members of $V$ is a finite open partition of $\beta_{0} X$, and clearly $W(\bar{V})=\overline{W(V)}$; as the $\overline{W(V)}$ 's form a fundamental system of entourages for the uniform structure of $\beta_{0} X, \beta_{0} X$ is ultraregular and hence ultranormal [1, Theorem 2], [21, 8-4]. Summarizing for ultraregular spaces, we have the following (essentially known) theorem:

Theorem 1. Let $X$ be an ultraregular space. Then $\beta_{0} X$ is an ultranormal compactification of $X$, clopen subsets of $X$ have clopen closures in $\beta_{0} X$, and for each ultraregular space $Y$ and each $f \in C(X, Y)$ there is a unique continuous function $\beta_{0} f: \beta_{0} X \rightarrow \beta_{0} Y$ extending $f$. If $X$ is ultranormal, then $B_{0}(X)=C^{*}(X, \mathbf{R})$ and hence $\beta_{\mathrm{o}} X=\beta X$; if $F$ is any ultraregular space containing at least two points, then $B_{0}(X)=C^{*}(X, F)$ and hence $\beta_{0} X=\beta_{F} X$. 
The definition of $\beta_{\mathrm{o}} X$ as the completion of $C^{*}(X, F)$ where $F$ is the discrete space of integers was first given in [23]. The Banaschewski compactification of $X$ has been extensively generalized. It is the $E$-compactification of $X$ where $E$ is a two-point set [10]. It is also an example of a Wallman compactification, introduced in [11] and generalized in [13].

Our purpose in this section is to show that $\beta_{\mathrm{o}} X$ may be identified with the set of characters of the $F$-algebra $C^{*}(X, F)$, where $F$ is an ultraregular topological field, and to obtain conditions on $X$ and $F$ sufficient for a description of $\beta_{0} X$ as the set of all maximal ideals of $C(X, F)$ (the analogue of the Gelfand-Kolmogoroff theorem [12, 7.3]) or the set of all $z$-ultrafilters (determined by $F$-valued continuous functions) on $X$ (the analogue of $[12,6.5]$ ).

Henceforth in this section, $F$ is an ultraregular topological field, $X$ an ultraregular space. For each $f \in C(X, F)$ we define $Z(f)$ to be $\{x \in X: f(x)$ $=0$ \}. An F-zeroset of $X$ is any subset of $X$ of the form $Z(f)$ where $f \in C(X, F)$.

LEMMA 1. If $m$ is a proper ideal of $C(X, F)$, then $\bigcap_{f \in m} \overline{Z(f)} \neq \varnothing$, where closure refers to $\beta_{\mathrm{o}} X$.

Proof. Suppose that $\bigcap_{f \in m} \overline{Z(f)}=\varnothing$. As $\beta_{\mathrm{o}} X$ is compact, there exist $f_{1}, \ldots, f_{n} \in \mathrm{m}$ such that $\bigcap_{i=1}^{n} \overline{Z\left(f_{i}\right)}=\varnothing$. Therefore the complements of $\overline{Z\left(f_{1}\right)}, \ldots, \overline{Z\left(f_{n}\right)}$ form an open cover of $\beta_{0} X$, so as $\beta_{0} X$ has zero Lebesgue covering dimension, there is a finite open partition $\left\{V_{1}, \ldots, V_{n}\right\}$ of $\beta_{0} X$ such that $V_{i} \subseteq \beta_{0} X-\overline{Z\left(f_{i}\right)}$ for all $i \in[1, n]$. Consequently, $C_{i}=V_{i} \cap X$ is a clopen subset of $X$ disjoint from $Z\left(f_{i}\right)$, so the function $g_{i}: X \rightarrow F$, defined by $g_{i}(x)=1 / f_{i}(x)$ if $x \in C_{i}, g_{i}(x)=0$ if $x \in X-C_{i}$, is continuous. Consequently, $1=\sum_{i=1}^{n} g_{i} f_{i} \in \mathrm{m}$, a contradiction.

TheOREM 2. Let $X$ be a compact, totally disconnected space. For each $a \in X$, let $\mathrm{m}_{a}=\{f \in C(X, F): f(a)=0\}$. Then $a \mapsto \mathrm{m}_{a}$ is a bijection from $X$ onto the set of all maximal ideals of $C(X, F)$.

The assertion follows from Lemma 1 (cf. $[12,7.2]$ ).

A character of an $F$-algebra $A$ is a nonzero homomorphism from the $F$-algebra $A$ into the $F$-algebra $F$.

Theorem 3. For each $a \in \beta_{0} X$, let $u_{a}: f \mapsto\left(\beta_{0} f\right)(a), f \in C^{*}(X, F)$. Then $a \mapsto u_{a}$ is a bijection from $\beta_{0} X$ onto the set $H$ of all characters of the F-algebra $C^{*}(X, F)$. Moreover, if $H$ is equipped with the weakest topology such that, for each $f \in C^{*}(X, F), u \mapsto u(f)$ is continuous from $H$ into $F$, then $a \mapsto u_{a}$ is a homeomorphism. Finally, $a \mapsto u_{a}^{-1}(0)$ is a bijection from $\beta_{0} X$ onto the set of all maximal ideals of $C^{*}(X, F)$. 
Proof. It is easy to verify that $f \mapsto \beta_{\mathrm{o}} f$ is an isomorphism from the $F$-algebra $C^{*}(X, F)$ onto the $F$-algebra $C\left(\beta_{\mathrm{o}} X, F\right)$. From this fact and Theorem 2 the assertion follows readily (cf. $[12,10.5]$ ).

To obtain meaningful analogues of the Gelfand-Kolmogoroff theorem and the description of $\beta X$ as the set of all $z$-ultrafilters, we need to know conditions implying that the intersection of two $F$-zerosets is an $F$-zeroset and that disjoint $F$-zerosets have disjoint closures in $\beta_{0} X$.

We shall say that a subset $A$ of $X$ is a $C_{\delta}$-set if $A$ is the intersection of a countable family of clopen subsets of $X$.

Theorem 4. If $F$ contains a sequence $\left(\lambda_{n}\right)_{n>0}$ of nonzero elements converging to zero, then the F-zerosets of $X$ are precisely the $C_{\delta}$-sets.

Proof. To show that an $F$-zeroset is a $C_{\delta}$-set, it suffices to find a sequence $\left(V_{n}\right)_{n>1}$ of clopen neighborhoods of zero in $F$ such that $\bigcap_{n=1}^{\infty} V_{n}=\{0\}$; for then, if $f \in C(X, F), Z(f)=\bigcap_{n=1}^{\infty} f^{-1}\left(V_{n}\right)$, a $C_{\delta}$-set. Let $V_{1}$ be a symmetric, clopen neighborhood of zero distinct from $F$. We define $\left(V_{n}\right)_{n \geqslant 1}$ inductively as follows: Given $V_{n}$, let $V_{n+1}$ be a symmetric clopen neighborhood of zero such that $V_{n+1}+V_{n+1} \subseteq V_{n}, V_{n+1} V_{n+1} \subseteq V_{n}$, and $V_{n+1} \subseteq \lambda_{0} V_{n} \cap \cdots \cap$ $\lambda_{n} V_{n}$. Then $\left(V_{n}\right)_{n>1}$ is a fundamental system of neighborhoods of zero for a ring topology $S$ on $F$. Indeed, we need only verify that, if $\beta \in F$ and if $n \geqslant 1$, there exists $p \geqslant 1$ such that $\beta V_{p} \subseteq V_{n}$. As $\lim _{k \rightarrow \infty} \beta \lambda_{k}=0$, there exists $m$ such that $\beta \lambda_{m} \in V_{n+1}$. Then $\beta V_{n+m+1} \subseteq V_{n}$, for $\beta V_{n+m+1} \subseteq$ $\beta \lambda_{m} V_{n+m} \subseteq V_{n+1} V_{n+m} \subseteq V_{n+1} V_{n+1} \subseteq V_{n}$. But a ring topology on the field $F$ is either Hausdorff or the trivial topology $\{F, \varnothing\}$ (for the closure of $\{0\}$ is an ideal). As $V_{1} \neq F$, therefore, $S$ is Hausdorff, so $\bigcap_{n=1}^{\infty} V_{n}=\{0\}$.

Conversely, let $A=\bigcap_{n=0}^{\infty} C_{n}$, where $\left(C_{n}\right)_{n \geq 0}$ is a decreasing sequence of clopen sets and $C_{0}=X$. The function $f: X \rightarrow F$, defined by $f(x)=\lambda_{n}$ for all $x \in C_{n}-C_{n+1}, n \geqslant 0$, and $f(x)=0$ for all $x \in A$, is easily seen to be continuous since $\lim _{n \rightarrow \infty} \lambda_{n}=0$, and clearly $Z(f)=A$.

THEOREM 5. Of the following conditions, if one of $1^{\circ}-3^{\circ}$ holds, then the intersection of two F-zerosets of $X$ is an F-zeroset. If $X$ is ultranormal, or if one of $1^{\circ}-5^{\circ}$ holds, then any two disjoint F-zerosets of $X$ have disjoint closures in $\beta_{0} X$.

$1^{\circ} F$ is not algebraically closed.

$2^{\circ} F$ possesses a sequence of nonzero elements converging to zero.

$3^{\circ} \mathrm{F}$ is discrete.

$4^{\circ} F$ is a proper subfield of an ultraregular topological field $K$.

$5^{\circ}$ The topology of $F$ is given by a valuation. 
Proof. If $X$ is ultranormal, then $\beta_{\mathrm{o}} X=\beta X$ by Theorem 1, and disjoint closed subsets of $X$ have disjoint closures in $\beta X$ since, by normality, they are contained in disjoint R-zerosets.

Assume that $1^{\circ}$ holds, and let $P=X^{n}+\alpha_{n-1} X^{n-1}+\cdots+\alpha_{1} X+\alpha_{0}$ be an irreducible polynomial of degree $n \geqslant 2$ over $F$. Let $f, g \in C(X, F)$, and let $h=f^{n}+\alpha_{n-1} f^{n-1} g+\cdots+\alpha_{1} f g^{n-1}+\alpha_{0} g^{n}$. Then $Z(h)=Z(f) \cap$ $Z(g)$, for if $g(x) \neq 0$ and if $\lambda=f(x) / g(x)$, then $h(x) / g(x)^{n}=P(\lambda) \neq 0$, so $h(x) \neq 0$, and if $g(x)=h(x)=0$, clearly also $f(x)=0$. Thus the intersection of two $F$-zerosets is an $F$-zeroset. Assume further that $Z(f) \cap Z(g)=\varnothing$. Then $h(x) \neq 0$ for all $x \in X$; thus if $k=f^{n} / h$, then $k(x)=0$ for all $x \in Z(f)$ and $k(x)=1$ for all $x \in Z(g)$; consequently, if $V$ is a clopen neighborhood of zero in $F$ that does not contain $1, k^{-1}(V)$ is a clopen neighborhood of $Z(f)$ disjoint from $Z(g)$; therefore by Theorem $1, \overline{Z(f)} \cap \overline{Z(g)}=\varnothing$.

Assume that $2^{\circ}$ holds. By Theorem 4, the intersection of two $F$-zerosets is an $F$-zeroset. Also by Theorem 4 , to prove that disjoint $F$-zerosets have disjoint closures in $\beta_{0} X$, it suffices by $1^{\circ}$ to find an ultraregular, metrizable, indiscrete field that is not algebraically closed. Such a field is the field $Q_{p}$ of $p$-adic numbers for any prime $p$, for by Eisenstein's criterion, $X^{n}-p$ is irreducible over $Q_{p}$ for all $n \geqslant 1$.

If $F$ is discrete, the $F$-zerosets are the clopen sets, and the assertions follow by Theorem 1 .

Assume that $4^{\circ}$ holds. By $1^{\circ}$, we may assume that $F$ is algebraically closed. Therefore $K$ contains an element $\lambda$ transcendental over $F$. The subfield $F(\lambda)$ is then an ultraregular topological field satisfying $1^{\circ}$, for $X^{2}-\lambda$ is irreducible over $F(\lambda)$. Since every $F$-zeroset of $X$ is clearly an $F(\lambda)$-zeroset, the assertion follows.

If $5^{\circ}$ holds, then so does $4^{\circ}$ by [7, Proposition 2, p. 161].

An F-z-ultrafilter, of course, is a maximal member of the class of all filter bases consisting of $F$-zerosets, ordered by inclusion. Statement $1^{\circ}$ of the following theorem is an ultraregular analogue of the Gelfand-Kolmogoroff theorem; statement $2^{\circ}$ provides an alternative description of $\beta_{0} X$, paralleling that given in $[12,6.5]$.

THEOREM 6. For each $a \in \beta_{\mathrm{o}} X$, let $\mathrm{m}_{a}=\{f \in C(X, F): a \in \overline{Z(f)}\}$ (where the closure is taken in $\beta_{0} X$ ), and let $U_{a}=\{Z: Z$ is an F-zeroset of $X$ and $a \in \bar{Z}$ \}.

$1^{\circ}$ If any two disjoint F-zerosets of $X$ have disjoint closures in $\beta_{0} X$ (see Theorem 5), then $a \mapsto m_{a}$ is a bijection from $\beta_{0} X$ onto the set of all maximal ideals of $C(X, F)$. 
$2^{\circ}$ If the intersection of any two F-zerosets of $X$ is an F-zeroset, and if any two disjoint F-zerosets of $X$ have disjoint closures in $\beta_{0} X$ (see Theorem 5), then $a \mapsto U_{a}$ is a bijection from $\beta_{0} X$ onto the set of all F-z-ultrafilters, and moreover, $\mathfrak{m} \longmapsto Z[\mathfrak{m}]=\{Z(f): f \in \mathfrak{m}\}$ is a bijection from the set of all maximal ideals of $C(X, F)$ onto the set of all $F$-z-ultrafilters.

Proof. We shall first show that if any two disjoint $F$-zerosets of $X$ have disjoint closures in $\beta_{0} X$, then $\overline{Z(f)} \cap \overline{Z(g)}=\overline{Z(f) \cap Z(g)}$ for all $f, g \in C(X, F)$. If $F$ is discrete, $Z(f)$ and $Z(g)$ are clopen sets; using the fact that $\overline{X-C}=$ $\beta_{\mathrm{o}} X-\bar{C}$ for any clopen subset $C$ of $X$ (Theorem 1 ), one may easily show that $\bar{A} \cap \bar{B}=\overline{A \cap B}$ for any clopen sets $A, B$ of $X$. Therefore we may assume that $F$ is not discrete, whence there exists $\gamma \in F$ unequal to 0 or 1 . Let $a \in \overline{Z(f)} \cap \overline{Z(g)}$, let $V$ be a clopen neighborhood of $a$ in $\beta_{0} X$, and let $W=X \cap V$. Let $U$ be a clopen neighborhood of 0 in $F$ such that $1 \notin U$. We define $f_{1}: X \rightarrow F$ by $f_{1}(x)=f(x)$ if $x \in f^{-1}(U), f_{1}(x)=\gamma$ if $x \in X-$ $f^{-1}(U)$. As $f^{-1}(U)$ is clopen, $f_{1}$ is continuous; clearly $Z(f)=Z\left(f_{1}\right)$. Let $f_{2}=f_{1}-\varphi$, where $\varphi$ is the characteristic function of $X-W$. One easily verifies that $f_{2} \in C(X, F)$ and that $Z\left(f_{2}\right)=Z(f) \cap W$. Similarly there exists $g_{2}$ $\in C(X, F)$ such that $Z\left(g_{2}\right)=Z(g) \cap W$. As $V$ is open, $a \in \overline{Z(f)} \cap V \subseteq$ $\overline{Z(f) \cap V}=\overline{Z(f) \cap W}=\overline{Z\left(f_{2}\right)}$, and similarly $a \in \overline{Z\left(g_{2}\right)}$. Therefore as disjoint $F$-zerosets have disjoint closures in $\beta_{\mathrm{o}} X$, we conclude that $\varnothing \neq Z\left(f_{2}\right) \cap Z\left(g_{2}\right)$ $\subseteq Z(f) \cap Z(g) \cap V$. Hence $a \in \overline{Z(f) \cap Z(g)}$.

For notational convenience, for each clopen subset $C$ of $\beta_{\mathrm{o}} X$ let $f_{C}$ be the restriction to $X$ of the characteristic function of $\beta_{\mathrm{o}} X-C$; thus $Z\left(f_{C}\right)=$ $X \cap C$, so $\overline{Z\left(f_{C}\right)}=C$. To prove $1^{\circ}$, we shall first show that $m_{a}$ is a proper ideal. If $f, g \in \mathfrak{m}_{a}$, then $a \in \overline{Z(f)} \cap \overline{Z(g)}=\overline{Z(f) \cap Z(g)} \subseteq \overline{Z(f+g)}$, so $f+$ $g \in \mathfrak{m}_{a}$, and if $h \in C(X, F)$, then $a \in \overline{Z(f)} \subseteq \overline{Z(f h)}$, so $f h \in \mathfrak{m}_{a}$. Clearly the constant function 1 does not belong to $\mathfrak{m}_{a}$. Hence $\mathfrak{m}_{a}$ is a proper ideal. Next, we shall prove that if $a \neq b$, then $\mathfrak{m}_{a} \nsubseteq \mathfrak{m}_{b}$. Indeed, let $C$ be a clopen subset of $\beta_{\mathrm{o}} X$ containing $a$ but not $b$; then clearly $f_{C} \in \mathrm{m}_{a}$ but $f_{C} \notin \mathrm{m}_{b}$. If $\mathfrak{m}$ is a maximal ideal of $C(X, F)$, by Lemma 1 there exists $a \in \beta_{\mathrm{o}} X$ such that $\mathfrak{m} \subseteq \mathfrak{m}_{a}$, whence $\mathfrak{m}=\mathfrak{m}_{a}$ as $\mathfrak{m}_{a}$ is a proper ideal. To complete the proof of $1^{\circ}$, therefore, we need only show that each $m_{a}$ is a maximal ideal. By Krull's theorem, there is a maximal ideal $m$ containing $\mathfrak{m}_{a}$, and by the preceding, $\mathfrak{m}=\mathfrak{m}_{b}$ for some $b \in \beta_{0} X$; thus $\mathfrak{m}_{a} \subseteq \mathfrak{m}_{b}$, so $a=b$ and $\mathfrak{m}_{a}=\mathfrak{m}_{b}=\mathfrak{m}$ as we saw above.

To prove $2^{\circ}$, we first note that by the first paragraph of the proof, $U_{a}$ is indeed a filter base of $F$-zerosets. Suppose that $U$ is a filter base of $F$-zerosets strictly containing $U_{a}$, and let $Z \in U$ be such that $Z \notin U_{a}$. Then $a \notin \bar{Z}$, 
so there is a clopen neighborhood $C$ of $a$ in $\beta_{0} X$ such that $C \cap \bar{Z}=\varnothing$. Consequently, $Z\left(f_{C}\right) \in U_{a}$, so $\varnothing=Z\left(f_{C}\right) \cap Z \in U$, a contradiction. Hence $U_{a}$ is an $F$-z-ultrafilter. For any clopen neighborhood $C$ of $a, Z\left(f_{C}\right) \in U_{a}$ and $Z\left(f_{C}\right) \subseteq C$, so $U_{a}$ converges to $a$; therefore $U_{a} \neq U_{b}$ if $a \neq b$. If $U$ is an $F$-z-ultrafilter on $X$, then $U$ has an adherent point $a \in \beta_{0} X$ as $\beta_{0} X$ is compact, so $a \in \bar{Z}$ for all $Z \in U$, whence $U \subseteq U_{a}$ and therefore $U=U_{a}$. The final assertion of $2^{\circ}$ follows from the fact that, for any $a \in \beta_{o} X, Z\left[\mathrm{~m}_{a}\right]=U_{a}$.

2. Repletions. Throughout this section, $F$ is a complete, separated uniform space, $X$ a Hausdorff topological space. We denote by $C(X, F)$ the weakest uniform structure on $X$ making each $f \in C(X, F)$ uniformly continuous. We call $X$ an F-replete space if $C(X, F)$ is complete and compatible with the topology of $X$; any $F$-replete space containing $X$ as a dense subset is called an F-repletion of $X$.

By [5, Proposition 8, p. 203, and Corollary, p. 210], $X$ is $F$-replete if and only if $X$ is homeomorphic to a closed subset of the cartesian product $F^{B}$ for some set $B$. Four consequences follow immediately: (1) Cartesian products and closed subspaces of $F$-replete spaces are $F$-replete. (2) The topological space $F$ is $F$-replete. (3) If $X$ is $F$-replete, then $X$ remains $F$-replete when the uniform structure of $F$ is replaced by any other compatible, complete uniform structure; thus $F$-repleteness does not depend on the given uniform structure of $F$, but only on its topology. (4) If $G$ is also a separated, complete uniform space and if the topological space $F$ is $G$-replete, then any $F$-replete space is also $G$-replete (for if $X$ is homeomorphic to a closed subset of $F^{A}$ and if $F$ is homeomorphic to a closed subset of $G^{B}$, then $X$ is homeomorphic to a closed subset of $G^{B \times A}$ ). In particular, if $F$ is $G$-replete and if $G$ is $F$-replete, then the $F$-replete and the $G$-replete spaces coincide.

The R-replete spaces are precisely the realcompact spaces; clearly also the $[0,1]$-replete spaces are the compact spaces, and the $\{0,1\}$-replete spaces are the compact ultraregular spaces. By (4), if $F$ is a realcompact topological space, then every $F$-replete space is realcompact.

The preceding remarks show that $X$ is $F$-replete if and only if $X$ is $F$-compact in the sense of Mrówka. The theory of $E$-compactness and $E$-compactifications, introduced in [10], has been developed in [18], [19], [4], [8], [9] and generalized in [14]. Our approach is less general than that of Mrówka since we require that $F$ admit a compatible, separated, complete uniform structure. However, in the only particular cases extensively explored, $F$ has a naturally associated complete uniform structure. More importantly, we believe that the use of uniform structures permits a more comprehensible discussion of $F$-repletions (i.e., $F$-compactifications). 
If $C(X, F)$ is compatible with the topology of $X$, we denote by $v_{F} X$ the completion of $X$ for $C(X, F)$. By [5, Proposition 14, p. 221], $C\left(v_{F} X, F\right)$ is the uniform structure of $v_{F} X$, so $v_{F} X$ is an $F$-repletion of $X$ to which each $f \in C(X, F)$ has a continuous extension. If $X$ is $F$-completely regular, we may identify $v_{F} X$ topologically with the subspace $X^{\prime}$ of $\beta_{F} X$ defined by $X^{\prime}=$ $\left\{x \in \beta_{F} X:\left(\beta_{F} f\right)(x) \in F\right.$ for all $\left.f \in C(X, F)\right\}$. Indeed, standard theorems about uniform spaces (especially [5, Proposition 9, p. 211 and Proposition 3, p. 206]) imply that $X^{\prime}$, equipped with the uniform structure $\mathcal{C}\left(X^{\prime}, F\right)$, is the completion of $X$ for $\mathcal{C}(X, F)$. If $F=\mathbf{R}$ and if $X$ is completely regular, the space $v_{F} X$, identified in this way with a subspace of $\beta X$, is the classical Hewitt realcompactification $v X$ of $X$. If $F$ and $X$ are ultraregular, we shall always consider $v_{F} X$ a subspace of $\beta_{0} X=\beta_{F} X$ by means of this identification; thus

$$
v_{F} X=\left\{x \in \beta_{0} X:\left(\beta_{0} f\right)(x) \in F \text { for all } f \in C(X, F)\right\} .
$$

A general study of extensions of a topological space, of which $F$-repletions form an example, has been undertaken in [17], [29], [15]. For example, the intersection of $F$-replete subsets of $X$ is $F$-replete [15, Lemma 2]; if $X$ is $F$-replete and if $f \in C(X, Y)$, then $f^{-1}(B)$ is $F$-replete for any $F$-replete subspace $B$ of $Y$ [15, Proposition 1]. Similarly, analogues of $[12,8.16,8.17]$ hold for $F$-replete spaces.

Let $f \in C(X, Y)$, where $Q(X, F)$ and $C(Y, F)$ are compatible with the topologies of $X$ and $Y$ respectively. One may easily show that $f$ is uniformly continuous for $C(X, F)$ and $C(Y, F)$, and consequently $f$ has a continuous extension $v_{F} f: v_{F} X \rightarrow v_{F} Y$. (If $X$ and $Y$ are $F$-completely regular, $v_{F} f$ is simply the function obtained by restricting the domain of $\beta_{F} f$ to $v_{F} X$ and its codomain to $v_{F} Y$.) In particular, every continuous function from $X$ into an $F$-replete space has a continuous extension to $v_{F} X$.

If $F$ is compact, then $C(X, F)=C^{*}(X, F)$, so $C(X, F)=C^{*}(X, F)$, and hence $v_{F} X=\beta_{F} X$. Thus $F$-repletions are primarily of interest only when the complete uniform space $F$ is not compact.

An important description of the realcompactification $v X$ of a completely regular space $X$ is that $v X=\left\{x \in \beta X\right.$ : for every sequence $\left(V_{n}\right)_{n>1}$ of neighborhoods of $x$ in $\beta X, \bigcap_{n=1}^{\infty} V_{n} \cap X \neq \varnothing$. Analogously, for any ultraregular space $X$, we define $v_{0} X$ to be the set of all $x \in \beta_{0} X$ such that for every sequence $\left(V_{n}\right)_{n>1}$ of neighborhoods of $x$ in $\beta_{0} X, \bigcap_{n=1}^{\infty} V_{n} \cap X \neq \varnothing$. We shall say that an ultraregular space $X$ is a $Q_{0}$-space if $X=v_{0} X$. (Another generalization of this description of a realcompactification is given in [29, Chapter III].)

We shall identify a class of complete uniform spaces, $F$, including the dis- 
crete space $\mathbf{N}$ of natural numbers, such that for every ultraregular space $X$, $v_{F} X=v_{0} X$. Even for the case $F=\mathrm{N}$, the most intensively studied case after realcompactifications, no information regarding repletions (i.e., $\mathbf{N}$-compactifications) has hitherto been available. This description yields at once the known result that every realcompact space that is strongly ultraregular in the following sense is $\mathbf{N}$-replete (no example of an $\mathbf{N}$-replete space that is not strongly ultraregular is known, and Roy's example [25], [26] of a realcompact ultraregular space $\Delta$ that is not ultranormal is known not to be N-replete [22]).

An ultraregular space $X$ is strongly ultraregular if $B_{0}(X)=C^{*}(X, \mathbf{R})$, whence $\beta_{0} X=\beta X$. By Theorem 1, every ultranormal space is strongly ultraregular. Conditions equivalent to strong ultraregularity are given in [22, Theorem 2].

Theorem 7. Let $X$ and $F$ be ultraregular. If $F$ is realcompact and strongly ultraregular, then $v_{0} X \subseteq v_{F} X$. If $F$ is not compact, then $v_{F} X \subseteq v_{0} X$.

Proof. Suppose that $F$ is realcompact and strongly ultraregular, and that $x_{\mathrm{o}} \in \beta_{\mathrm{o}} X-v_{F} X$. Then there exists $f \in C(X, F)$ such that $\left(\beta_{\mathrm{o}} f\right)\left(x_{\mathrm{o}}\right) \in \beta_{\mathrm{o}} F-$ $F=\beta F-F$. Thus as $F$ is realcompact, there is a decreasing sequence $\left(V_{n}\right)_{n \geq 1}$ of open neighborhoods of $\left(\beta_{0} f\right)\left(x_{0}\right)$ such that $\bigcap_{n=1}^{\infty} V_{n} \cap F=\varnothing$. Then $W_{n}=$ $\left(\beta_{0} f\right)^{-1}\left(V_{n}\right)$ is an open neighborhood of $x_{0}$ in $\beta_{0} X$ for all $n \geqslant 1$, and $\bigcap_{n=1}^{\infty} w_{n} \cap X=\varnothing$, so $x_{0} \in \beta_{0} X-v_{0} X$.

Next, suppose that $F$ is not compact and that $x_{0} \in \beta_{0} X-v_{0} X$. As $\beta_{0} X$ is ultraregular, there is a decreasing sequence $\left(C_{n}\right)_{n} \geq 0$ of clopen neighborhoods of $x_{0}$ such that $C_{0}=\beta_{\mathrm{o}} X$ and $\bigcap_{n=0}^{\infty} C_{n} \cap X=\varnothing$. As $F$ is not precompact, there is an entourage $U$ for which no finite cover of $F$ by $U$-small sets exists. Let $V$ be a symmetric entourage such that $V^{2} \subseteq U$. Then for each $x \in X$, $V(x)$ is $U$-small, so there exists a sequence $\left(a_{i}\right)_{i>0}$ such that $a_{i} \in F-\bigcup_{j<i} V\left(a_{j}\right)$ for all $i \geqslant 1$. Let $f: X \rightarrow F$ be defined by $f(x)=a_{i}$ for all $x \in X \cap$ $\left(C_{i}-C_{i+1}\right), i \geqslant 0$. Then as $X \cap\left(C_{i}-C_{i+1}\right)$ is open in $X, f$ is continuous. Let $D=\left\{a_{i}: i \geqslant 0\right\}$, which is clearly a closed, discrete subspace of $F$, and let $g$ be the continuous extension of $f$ to $v_{F} X$. Then $g\left(v_{F} X\right) \subseteq D$ since $D$ is closed in $F, X$ is dense in $v_{F} X$, and $g(X) \subseteq D$. Suppose that $x_{\mathrm{o}} \in v_{F} X$. Then $g\left(x_{0}\right)=a_{r}$ for some $r \geqslant 0$; as $\left\{a_{r}\right\}$ is open in $D, U=g^{-1}\left(a_{r}\right)$ is an open neighborhood of $x_{0}$ in $v_{F} X$. Now $U \cap C_{r+1}$ is an open neighborhood of $x_{0}$ in $v_{F} X$, so there exists $x \in U \cap C_{r+1} \cap X$; thus $f(x)=g(x)=a_{r}$ as $x \in U$, but $f(x) \in\left\{a_{i}: i>r\right\}$ as $x \in C_{r+1} \cap X$, a contradiction. Hence $x_{\mathrm{o}} \notin v_{F} X$.

The first assertion of the following theorem is evident; the second follows from Theorem 7. 
THEOREM 8. An ultraregular Lindelöf space is a $Q_{0}$-space. If $F$ is ultraregular but not compact, every ultraregular Lindelöf space is F-replete.

The second assertion of the following theorem was proved by different methods in [14, Theorem 6.4] and [22].

THEOREM 9. Let $F$ be a realcompact, strongly ultraregular, noncompact space (as $F$ is realcompact, $F$ admits a compatible complete uniform structure, namely, $C(F, \mathbf{R})$ ).

$1^{\circ}$ For any ultraregular space $X, v_{F} X=v_{0} X=v_{\mathrm{N}} X$.

$2^{\circ} F$ is $\mathrm{N}$-replete, and $\mathrm{N}$ is F-replete.

PRoof. Since $\mathbf{N}$ is realcompact, strongly ultraregular, but not compact, $1^{\circ}$ follows from Theorem 7. As remarked earlier, $F$ is $F$-replete and $\mathrm{N}$ is $\mathrm{N}$-replete; hence by $1^{\circ}, F=v_{F} F=v_{\mathrm{N}} F$, so $F$ is $\mathrm{N}$-replete, and $\mathrm{N}=v_{\mathrm{N}} \mathrm{N}=v_{F} \mathrm{~N}$, so $\mathbf{N}$ is $F$-replete (Theorem 8 also implies that $\mathbf{N}$ is $F$-replete).

For example, if $\Delta$ is Roy's space, then $v_{F} \Delta=v_{0} \Delta=v_{N} \Delta \neq \Delta=v \Delta$.

3. Repletions relative to complete topological fields. Throughout this section, $F$ is a complete Hausdorff topological field.

TheOREM 10. Let $X$ and $F$ be ultraregular. For each $a \in v_{F} X$, let $u_{a}$ : $f \mapsto\left(v_{F} f\right)(a), f \in C(X, F)$. Then $a \mapsto u_{a}$ is a homeomorphism from $v_{F} X$ onto the set $H$ of all characters of the F-algebra $C(X, F)$, equipped with the weakest topology such that for each $f \in C(X, F), u \mapsto u(f)$ is continuous from $H$ into $F$.

PRoof. It is easy to verify that $u_{a} \in H$. To show that $a \mapsto u_{a}$ is surjective, let $u \in H$, and let $\mathfrak{m}=\operatorname{ker} u$. By Lemma 1 , there exists $a \in \beta_{0} X$ such that $\left(\beta_{\mathrm{o}} f\right)(a)=0$ for all $f \in \mathfrak{m}$. Given $f \in C(X, F)$, let $\lambda=u(f)$, and let $g=f-\lambda .1$. Then $u(g)=0$, so $\left(\beta_{\mathrm{o}} g\right)(a)_{0}=0$. Let $F$ be a filter on $X$ converging to $a$; then $\lim _{F} g=\left(\beta_{0} g\right)(a)=0$, so $\left(\beta_{0} f\right)(a)=\lim _{F} f=\lim _{F}(\lambda .1+g)$ $=\lambda=u(f) \in F$. Consequently, $a \in v_{F} X$. Thus $\operatorname{ker} u \subseteq \operatorname{ker} u_{a}$, so $u=u_{a}$ as both are homomorphisms whose kernels have codimension 1 . The remaining verifications are easy.

The following corollaries are analogues of the original characterizations of realcompact spaces and realcompactifications in terms of maximal ideals of $C(X, \mathbf{R})$.

COROLlaRY 1. If $X$ and $F$ are ultraregular, then $X$ is F-replete if and only if for each character $u$ of $C(X, F)$ [for each maximal ideal $\mathfrak{m}$ of $C(X, F)$ of codimension one] there exists $a \in X$ such that $u(f)=f(a)[f(a)=0]$ for all $f \in C(X, F)$ [for all $f \in \mathfrak{m}$ ]. 
Corollary 2. Let $X$ and $F$ be ultraregular. If any two disjoint F-zerosets, of $X$ have disjoint closures in $\beta_{\mathrm{o}} X$ (see Theorem 5), then the bijection $a \mapsto m_{a}$ of $1^{\circ}$ of Theorem 6 takes $v_{F} X$ onto the set of all maximal ideals of $C(X, F)$ of codimension one.

Proof. First we observe that if $a \in v_{F} X$, then $\mathrm{m}_{a}=\operatorname{ker} u_{a}$. Indeed, as both $m_{a}$ and ker $u_{a}$ are maximal ideals, it suffices to prove that $m_{a} \subseteq \operatorname{ker} u_{a}$; but if $f \in \mathrm{m}_{a}$, then $a \in \overline{Z(f)}$, so $\left(v_{F} f\right)(a)=0$, whence $u_{a}(f)=0$. In particular, if $a \in v_{F} X$. then $\mathfrak{m}_{a}$ has codimension one. Conversely, let $m$ be a maximal ideal having codimension one. Then $\mathrm{m}=\operatorname{ker} u$ for some $u \in H$; by Theorem 10, $u=u_{a}$ for some $a \in v_{F} X$, so $\mathrm{m}=\operatorname{ker} u=\operatorname{ker} u_{a}=\mathrm{m}_{a}$.

THEOREM 11. Let $X$ and $F$ be ultraregular, and let $F$ either possess $a$ sequence of nonzero elements converging to zero or be discrete. The bijection $a \mapsto U_{a}$ of $2^{\circ}$ of Theorem 6 takes $v_{0} X$ onto the set of all F-z-ultrafilters that are closed under countable intersection.

Proof. If $a \notin v_{0} X$, then there exists a sequence $\left(V_{n}\right)_{n}>_{1}$ of clopen neighborhoods of $a$ in $\beta_{0} X$ such that $\bigcap_{n=1}^{\infty} V_{n} \cap X=\varnothing$. As $V_{n}$ is open, $a \in$ $V_{n} \subseteq \overline{V_{n} \cap X}$, so as $V_{n} \cap X$ is an $F$-zeroset, $V_{n} \cap X \in U_{a}$. Consequently, $\left(V_{n} \cap X\right)_{n>1}$ is a sequence of members of $U_{a}$ whose intersection does not belong to $U_{a}$.

Conversely, let $a \in v_{0} X$. We shall first show that if $\left(W_{n}\right)_{n \geq 1}$ is any sequence of members of $u_{a}$, then $\bigcap_{n=1}^{\infty} w_{n} \neq \varnothing$. Indeed, for each $n \geqslant 1$ there exists, by Theorem 4, a sequence $\left(V_{n, m}\right)_{m} \geqslant 1$ of clopen sets of $X$ such that $W_{n}=\bigcap_{m=1}^{\infty} V_{n, m}$. As $a \in \bar{W}_{n} \subseteq \bar{V}_{n, m}, \bar{V}_{n, m}$ is a clopen neighborhood of $a$ in $\beta_{0} X$ and $\bar{V}_{n, m} \cap X=V_{n, m}$. Thus

$$
\bigcap_{n=1}^{\infty} w_{n}=\bigcap_{n, m=1}^{\infty}\left(\bar{V}_{n, m} \cap X\right)=\bigcap_{n, m=1}^{\infty} \bar{V}_{n, m} \cap X \neq \varnothing
$$

by the definition of $v_{0} X$. Next, let $\left(Z_{n}\right)_{n \geq 1}$ be any sequence of members of $U_{a}$, and let $Z=\bigcap_{n=1}^{\infty} Z_{n}$. For any $W \in U_{a}, Z \cap W \neq \varnothing$ by the preceding, since each $Z_{n} \cap W \in U_{a}$. Hence $U=\{U: U$ is an $F$-zeroset of $X$ containing $Z \cap W$ for some $\left.W \in U_{a}\right\}$ is an $F$-z-ultrafilter containing $U_{a}$. Therefore $U=$ $U_{a}$, so $Z=Z \cap X \in U_{a}$.

We are particularly interested in the case where the topology of $F$ is either ultrametrizable or given by a valuation. (A metric $d$ on $X$ is an ultrametric if $d(x, z) \leqslant \max \{d(x, y), d(y, z)\}$ for all $x, y, z \in X$.) In both cases there is a fundamental system of idempotent symmetric entourages for the associated uniform structure of $F$ that is totally ordered by inclusion (an entourage $U$ is 
idempotent if $U \circ U=U$ ). Consequently, $F$ and all of its subsets are ultranormal (and hence strongly ultraregular) by the following theorem.

THEOREM 12. If $F$ is a separated uniform space for which there is a fundamental system $U$ of symmetric idempotent entourages that is totally ordered by inclusion, then $F$ is ultranormal.

Proof. We may assume that $F$ is not discrete, so that $U$ has no smallest member. The Dedekind completion of the totally ordered set $U$ may be explicitly constructed as a subset of the entourages of $F$. Namely, let $\Phi$ be the set of all subfamilies $F$ of $U$ having the following two properties: there exists $V$ $\in U$ such that every member of $F$ contains $V$; and if $U \in F$ and if $W \in U$ is an entourage containing $U$, then $W \in F$. Let $U^{*}=\{\bigcap F: F \in \Phi\}$. Clearly $U^{*}$ is a set of symmetric idempotent entourages that contains $U$ and is totally ordered by inclusion, and every family $G$ of members of $U^{*}$ that is bounded below (i.e., for which there exists $V \in U$ contained in each member of $G$ ) admits an infimum in $U^{*}$ (namely, $\cap G$ ). Adjoining the diagonal subset $\Delta$ of $F \times F$ to $U^{*}$, which is smaller than each member of $U^{*}$ for the ordering $\subseteq$, we may therefore define a "generalized ultrametric" $d$ on $F \times F$ by $d(x, y)=$ the infimum (in $U^{*} U\{\Delta\}$ ) of all $U \in U^{*}$ such that $(x, y) \in U$. One easily shows that the uniform structure defined in an obvious way by $d$ is the given uniform structure. For each subset $A$ of $F$ and each $x \in F$, let $d(x, A)=$ $\inf \{d(x, a): a \in A\}$. One easily establishes that $d(x, A)=\Delta$ if and only if $x \in \bar{A}$ and that $d(x, A)=d(y, A)$ whenever $d(x, y) \subset d(x, A)$. From these facts one may show that if $A$ and $B$ are disjoint closed subsets of $F$, then $C=\{x \in F: d(x, A) \subset d(x, B)\}$ is a clopen set containing $A$ and disjoint from $B$.

To apply the results of $\S 2$ to the case where $F$ is a complete topological field whose topology is either ultrametrizable or given by a valuation, we need to determine necessary and sufficient conditions for $F$ to be realcompact. This leads to the problem of measurable cardinals (a cardinal number $m$ is measurable if there is a countably additive function $\mu$ from the set of all subsets of some set $X$ of cardinality $m$ into $\{0,1\}$ such that $\mu(X)=1$ and $\mu(\{x\})=0$ for all $x \in X$; the existence of measurable cardinals cannot be proved from the usual axioms of set theory). Indeed, it is easy to show that the cardinality $|X|$ of a set $X$ is nonmeasurable if and only if the discrete topological space $X$ is a $Q_{0}$-space. By a theorem of Shirota [27], $[12,15.20]$, a completely regular space $X$ is realcompact if and only if $X$ admits a compatible complete uniform structure and the cardinality of every closed, discrete subset of $X$ is nonmeasurable In particular, if $X$ is a separated uniform space such that $|X|$ is nonmeasurable, then its completion $X^{\wedge}$ is realcompact (for as $X^{\wedge}$ may be identified with a 
family of Cauchy filters on $X,\left|X^{\wedge}\right| \leqslant 2^{2}|X|$, and hence $\left|X^{\wedge}\right|$ is also nonmeasurable).

In the cases under consideration, the condition that $|F|$ be nonmeasurable is necessary as well as sufficient for $F$ to be realcompact, as the following two theorems show.

THEOREM 13. An ultrametric space $F$ is realcompact if and only if $|F|$ is nonmeasurable.

Proof. The condition is sufficient, for as noted above, the completion of $F$ is realcompact, whence $F$ is by $[12,8.15]$. Necessity: Let $\left(U_{n}\right)_{n>1}$ be a fundamental system of idempotent entourages, and for each $n \geqslant 1$ let $D_{n}$ be a subset of $F$ consisting of one point from each member of the open partition $V_{n}=\left\{U_{n}(x): x \in F\right\}$. Then $D_{n}$ is a closed discrete set, so $\left|V_{n}\right|=\left|D_{n}\right|$ is nonmeasurable. Since $x \mapsto\left(U_{n}(x)\right)_{n>1}$ is an injection from $F$ into $\Pi_{n=1}^{\infty} V_{n},|F| \leqslant$ $\aleph_{0}\left(\sup _{n}\left|V_{n}\right|\right)$, so $|F|$ is nonmeasurable.

THEOREM 14. Let $F$ be a topological field whose topology is given by a valuation $v$, and let $k$ be the residue field of $F$. The following statements are equivalent:

$1^{\circ} F$ is realcompact.

$2^{\circ}|F|$ is nonmeasurable.

$3^{\circ}$ Every subset of $F$ is realcompact.

$4^{\circ}|k|$ is nonmeasurable.

Proof. Let $\Gamma$ be the value group of $v$, let $\Gamma_{+}$be the set of positive elements of $\Gamma$, and let $A$ be the valuation ring of $v$. Assume $1^{\circ}$. For each $\alpha \in \Gamma_{+}$, let $V_{\alpha}$ be the open partition $\left\{V_{\alpha}(x): x \in A\right\}$ of $A$ determined by $\alpha$, where $V_{\alpha}(x)=\{y \in A: v(x-y)>\alpha\}$. A set $D_{\alpha}$ consisting of one point in each member of $V_{\alpha}$ is then discrete and closed, so $\left|V_{\alpha}\right|=\left|D_{\alpha}\right|$ is nonmeasurable. In particular, the canonical epimorphism from $A$ onto $k$ takes $D_{\alpha}$ onto $k$, so $|k|$ is nonmeasurable. As $v$ determines a bijection from $k$ onto $\Gamma_{+} \cup\{+\infty\}$, $\left|\Gamma_{+}\right|$is also nonmeasurable. Therefore as $x \mapsto\left(V_{\alpha}(x)\right)_{\alpha \in \Gamma_{+}}$is an injection from $A$ into $\Pi_{\alpha \in \Gamma_{+}} V_{\alpha},|A| \leqslant\left|\Gamma_{+}\right|\left(\sup \left|V_{\alpha}\right|\right)$, and hence $|A|$ is nonmeasurable. Therefore $|F| \leqslant 2|A|$ is nonmeasurable.

Next, assume $2^{\circ}$. As noted above, $\left|F^{\wedge}\right|$ is nonmeasurable. Moreover, the multiplicative group $F^{\wedge}-\{0\}$ of nonzero elements of $F^{\wedge}$ is complete for its associated uniform structure [6, Proposition 8, p. 85], and $F^{\wedge}-\{0\}$ is therefore realcompact by Shirota's theorem. For each $a \in F^{\wedge}, F^{\wedge}-\{a\}$ is homeomorphic to $F^{\wedge}-\{0\}$ and hence is also realcompact. Therefore every subset of $F^{\wedge}$ is realcompact $[12,8.17]$, and $3^{\circ}$ holds. By Krull's lemma $[24$, p. 80$]$, 
$|F| \leqslant|k|^{|\Gamma|}$, and hence as $|\Gamma| \leqslant 2\left|\Gamma_{+}\right| \leqslant 2|k|, 4^{\circ}$ implies $2^{\circ}$.

Applying Theorems 5, 6, 9, Corollary 2 of Theorem 10, and Theorems 1114 , and recalling that a compact field is finite, we obtain the following summary:

THEOREM 15. Let $F$ be an infinite, complete topological field whose topology is either ultrametrizable or defined by a valuation and whose cardinality $|F|$ is nonmeasurable, and let $X$ be an ultraregular space.

$1^{\circ}$ Every subset of $F$ is ultranormal, realcompact, and F-replete.

$2^{\circ}$ If $X$ is F-replete, then $X$ is realcompact.

$3^{\circ} v_{F} X=v_{0} X$, so $X$ is F-replete if and only if $X$ is a $Q_{0}$-space.

$4^{\circ}$ If $X$ is ultranormal, then $v_{F} X=v_{0} X=v X$, so $X$ is F-replete if and only if $X$ is realcompact.

$5^{\circ}$ The function $a \mapsto \mathrm{m}_{a}=\{f \in C(X, F): a \in \overline{Z(f)}\}$ is a bijection from $\beta_{0} X$ onto the set of all maximal ideals of $C(X, F)$, and takes $v_{F} X$ onto the set of all maximal ideals of codimension one. Thus $X$ is compact if and only if each maximal ideal of $C(X, F)$ is fixed, and $X$ is F-replete if and only if each maximal ideal of $C(X, F)$ of codimension one is fixed.

$6^{\circ}$ If $F$ is ultrametrizable, then the function $a \mapsto U_{a}=\{Z: Z$ is an $F$-zeroset of $X$, and $a \in \bar{Z}$ \} is a bijection from $\beta_{0} X$ onto the set of all F-z-ultrafilters, and takes $v_{F} X$ onto the set of all $F$-z-ultrafilters that are closed under countable intersection.

The immanence of the problem of measurable cardinals in the theory of repletions is further indicated by the following theorem.

THEOREM 16. Let $F$ be an infinite, complete, ultraregular topological field such that $|F|$ is nonmeasurable, let $X$ be ultraregular, and let $\left(X_{\alpha}\right)_{\alpha \in A}$ be an open partition of $X$. Then $X$ is F-replete if and only if $|A|$ is nonmeasurable and each $X_{\alpha}$ is F-replete.

Proof. Necessity: Each $X_{\alpha}$ is closed and hence is also $F$-replete. Let $D$ be a set consisting of one element from each $X_{\alpha}$. Then $D$ is closed and discrete, so since $X$ is realcompact, $|A|=|D|$ is nonmeasurable.

Sufficiency: For each $f \in C(X, F)$ and each $\alpha \in A$, let $f_{\alpha}$ be the restriction of $f$ to $X_{\alpha}$. As $\left(X_{\alpha}\right)$ is an open partition of $X, J: f \mapsto\left(f_{\alpha}\right)$ is an isomorphism from the $F$-algebra $C(X, F)$ onto the $F$-algebra $\Pi_{\alpha \in A} C\left(X_{\alpha}, F\right)$. Let $w$ be a character of $C(X, F)$, and let $u=w^{\circ} J^{-1}$. By a theorem of Białynicki-Birula and Żelazko [3], [31], there exist $\beta \in A$ and a character $v$ of $C\left(X_{\beta}, F\right)$ such that $u=v \circ \mathrm{pr}_{\beta}$, where $\mathrm{pr}_{\beta}$ denotes the projection from $\Pi_{\alpha \in A} C\left(X_{\alpha}, F\right)$ onto $C\left(X_{\beta}, F\right)$. By Theorem 10, there exists $b \in X_{\beta}$ such that $v(h)=h(b)$ for all $h \in C\left(X_{\beta}, F\right)$. Hence for each $f \in C(X, F), w(f)=$ 
$\left(\nu \circ \mathrm{pr}_{\beta} \circ J(f)=v\left(f_{\beta}\right)=f_{\beta}(b)=f(b)\right.$. Therefore, by Theorem 10,X is $F$-replete.

The same proof establishes the corresponding result for realcompact spaces:

If $\left(X_{\alpha}\right)_{\alpha \in A}$ is an open partition of a completely regular space $X$, then $X$ is realcompact if and only if $|A|$ is nonmeasurable and each $X_{\alpha}$ is realcompact.

The first examples of nonrealcompact completely regular spaces were noncompact pseudocompact spaces. We recall that a subset $B$ of a topological field $F$ is bounded if for every neighborhood $V$ of zero there is a neighborhood $U$ of zero such that $B U \subseteq V$; an $F$-valued function is bounded if its range is a bounded subset of $F$. The obvious generalization of the definition of pseudocompactness is the following.

Definition. A Hausdorff space $X$ is F-pseudocompact if $C(X, F)$ is compatible with the topology of $X$ and if every continuous function from $X$ into $F$ is bounded.

If $F$ is a locally compact field, then the bounded subsets of $F$ coincide with the relatively compact ones, and consequently if $X$ is $F$-pseudocompact, then $C^{*}(X, F)=C(X, F)$. This holds much more generally, as the following two theorems show.

THEOREM 17. Let $F$ be a complete, strongly ultraregular, realcompact topological field that contains an unbounded sequence $\left(\beta_{n}\right)_{n \geq 1}$, and let $X$ be an ultraregular space. The following statements are equivalent:

$1^{\circ} X$ is.F-pseudocompact.

$2^{\circ} v_{0} X=\beta_{0} X$.

$3^{\circ} v_{F} X=\beta_{0} X$.

$4^{\circ} C(X, F)=C^{*}(X, F)$.

Proof. To show that $1^{\circ}$ implies $2^{\circ}$, let $a \in \beta_{0} X$, and let $\left(V_{n}\right)_{n>1}$ be any decreasing sequence of clopen neighborhoods of $a$ in $\beta_{0} X$ such that $V_{1}=$ $\beta_{0} X$. Suppose that $\bigcap_{n=1}^{\infty} V_{n} \cap X=\varnothing$. Then since $V_{n} \cap X \neq \varnothing$ for all $n \geqslant 1$, we may assume that $\left(V_{n} \cap X\right)_{n \geqslant 1}$ is a strictly decreasing sequence of clopen subsets of $X$. The function $f: X \rightarrow F$, defined by $f(x)=\beta_{n}$ for all $x \in$ $\left(V_{n}-V_{n+1}\right) \cap X$, is an unbounded, continuous function, a contradiction. Consequently, $a \in v_{0} X$.

By $1^{\circ}$ of Theorem $9,2^{\circ}$ implies $3^{\circ}$. Since each $f \in C(X, F)$ has a continuous extension to $v_{F} X, 3^{\circ}$ implies $4^{\circ}$. Since a relatively compact subset of $F$ is bounded, $4^{\circ}$ implies $1^{\circ}$.

THEOREM 18. Let $F$ be a Hausdorff topological field. (1) If $F$ possesses a sequence of nonzero elements converging to zero, then $F$ possesses an unbounded sequence. (2) If the topology of $F$ is given by a proper valuation $v$, then $F$ has an unbounded sequence if and only if $F$ is metrizable. 
Proof. (1) Let $V$ be a neighborhood of zero such that $1 \notin V$, and let $\left(\lambda_{n}\right)_{n>1}$ be a sequence of nonzero elements converging to zero. Let $\beta_{n}=\lambda_{n}^{-1}$. Suppose that $\left(\beta_{n}\right)_{n>1}$ were bounded. Then there would exist a neighborhood $U$ of zero such that $\left(\beta_{n}\right) U \subseteq V$. Let $m$ be such that $\lambda_{m} \in U$; then $1=$ $\beta_{m} \lambda_{m} \in V$, a contradiction.

(2) Let $\Gamma$ be the value group of $v$. A subset $B$ of $F$ is bounded if and only if $v(B)$ has a lower bound in $\Gamma$. Consequently, if $\left(\lambda_{n}\right)_{n \geq 1}$ is a sequence of nonzero elements, then $\lim _{n \rightarrow \infty} \lambda_{n}=0$ if and only if $\left(\lambda_{n}^{-1}\right)_{n>1}$ is unbounded; but if $\lim _{n \rightarrow \infty} \lambda_{n}=0$, then $\left(\lambda_{n} A\right)_{n>1}$ is a fundamental system of neighborhoods of zero, where $A$ is the valuation ring, so $F$ is metrizable.

In contrast, let $F$ be a complete field whose topology is given by a proper valuation $v$ but is not metrizable (equivalently, the value group of $v$ has no cofinal sequence). Then every $\sigma$-compact ultraregular space $X$ is $F$-replete and $F$-pseudocompact. Indeed, if $X=\bigcup_{n=1}^{\infty} K_{n}$, where each $K_{n}$ is compact, then $X$ is $F$-replete by Theorem 8 and $3^{\circ}$ of Theorem 15; if $f \in C(X, F)$, then for each $n \geqslant 1$ there is a lower bound $\alpha_{n}$ of $v\left(f\left(K_{n}\right)\right)$ since $f\left(K_{n}\right)$ is compact and hence bounded, so a lower bound of $\left(\alpha_{n}\right)_{n \geq 1}$ is a lower bound of $v(f(X))$.

4. Measure-theoretic interpretations. Throughout this section, $X$ is an ultraregular space, and $F$ is a complete, Hausdorff topological field for which the set $V$ of open additive subgroups is a fundamental system of neighborhoods of zero.

The topology $T_{u}$ of uniform convergence on $C^{*}(X, F)$ is easily seen to make $C^{*}(X, F)$ a topological $F$-algebra (to verify that for each $f \in C^{*}(X, F)$, $g \mapsto g f$ and $\lambda \mapsto \lambda f$ are continuous at zero, recall that $f(X)$ is relatively compact and hence bounded in $F)$. For each $V \in V$, let $V_{u}=\left\{f \in C^{*}(X, F)\right.$ : $f(X) \subseteq V\}$; then $V_{u}=\left\{V_{u}: V \in V\right\}$ is a fundamental system of neighborhoods of zero in $C^{*}(X, F)$ for $T_{u}$. We shall denote by $C^{*}(X, F)^{d}$ the topological dual of $C^{*}(X, F)$, equipped with the topology $T_{u}$.

We denote by $S(X)$ the algebra of all clopen subsets of $X$, and by $M(X, F)$ the set of all bounded, finitely additive functions from $S(X)$ into $F$. A finitely additive $F$-valued function $\mu$ defined on an algebra $S$ of sets is monotone if for all $S, S^{\prime} \in S$, if $\mu(S)=0$ and if $S^{\prime} \subseteq S$, then $\mu\left(S^{\prime}\right)=0$. We denote by $M_{\mathrm{o}}(X, F)$ the set of all monotone members of $M(X, F)$ whose range is $\{0,1\}$. (If the characteristic of $F$ is not 2, every member of $M(X, F)$ whose range is $\{0,1\}$ is monotone.) We recall (Theorem 3$)$ that there is a bijection $a \mapsto u_{a}$ from $\beta_{0} X$ onto the set $H(X, F)$ of all characters of the $F$-algebra $C^{*}(X, F)$, where $u_{a}(f)=\left(\beta_{o} f\right)(a)$ for all $f \in C^{*}(X, F)$.

For each $v \in C^{*}(X, F)^{d}$, we denote by $\mu_{\nu}$ the function from $S(X)$ into 
$F$ defined by $\mu_{\nu}(S)=v\left(\varphi_{S}\right)$ for all $S \in S(X)$, where $\varphi_{S}$ is the $F$-valued characteristic function of $S$.

TheOREM 19. (1) $\nu \mapsto \mu_{\nu}$ is a bijection from $C^{*}(X, F)^{d}$ onto $M(X, F)$. (2) $H(X, F) \subseteq C^{*}(X, F)^{d}$, i.e., every character of $C^{*}(X, F)$ is continuous for $T_{u}$. (3) $v \in H(X, F)$ if and only if $\mu_{\nu} \in M_{0}(X, F)$.

Proof. (1) Clearly $\mu_{\nu}$ is a finitely additive function. We shall show that the range $B_{v}$ of $\mu_{\nu}$ is bounded. Since every subset of a discrete field is bounded, we shall assume that $F$ is not discrete. Let $V \in V$; there exists $U \in V$ such that $U U \subseteq V$. As $v$ is continuous, there exists $W \in V$ such that $v\left(W_{u}\right) \subseteq U$. Let $\alpha$ be a nonzero member of $W$. Then for any $S \in S(X), \alpha \varphi_{S}$ $\in W_{u}$, so $\mu_{v}(S) \cdot(\alpha U)=v\left(\alpha \varphi_{S}\right) \cdot U \subseteq U U \subseteq V$; thus $B_{v}(\alpha U) \subseteq V$.

To complete the proof of (1), we shall show that if $\mu \in M(X, F)$, then there is a unique $\nu \in C^{*}(X, F)^{d}$ such that $\mu_{\nu}=\mu$. Let $D(X, F)$ be the algebra of all continuous $F$-valued functions on $X$ with finite range. Each member of $D(X, F)$ is uniquely of the form $\sum_{i=1}^{n} \lambda_{i} \varphi_{S_{i}}$ where $\left\{S_{1}, \cdots, S_{n}\right\}$ is an open partition of $X$ and $\left(\lambda_{i}\right)_{1<i<n}$ is a sequence of distinct scalars. We define $v: D(X, F) \rightarrow F$ by

$$
v\left(\sum_{i=1}^{n} \lambda_{i} \varphi_{S_{i}}\right)=\sum_{i=1}^{n} \lambda_{i} \mu\left(S_{i}\right)
$$

It is easy to verify that $\nu$ is a linear form on $D(X, F)$ and, indeed, is the only linear form such that $v\left(\varphi_{S}\right)=\mu(S)$ for all $S \in S(X)$. To show that $v$ is continuous, let $V \in V$, and let $W \in V$ be such that $W B \subseteq V$, where $B$ is the range of $\mu$. If $\left\{S_{1}, \cdots, S_{n}\right\}$ is an open partition of $X$ and if $\left(\lambda_{i}\right)_{1<i<n}$ is a sequence of scalars such that $\sum_{i=1}^{n} \lambda_{i} \varphi_{S_{i}} \in W_{u}$, then

$$
v\left(\sum_{i=1}^{n} \lambda_{i} \varphi_{S_{i}}\right)=\sum_{i=1}^{n} \lambda_{i} \mu\left(S_{i}\right) \in n . W B \subseteq n \cdot V=V
$$

as $V$ is a subgroup. Thus $v$ is continuous on $D(X, F)$. Now $D(X, F)$ is dense in $C^{*}(X, F)$. To see this, let $f \in C^{*}(X, F)$, and let $V \in V$. As $f(X)$ is relatively compact, there exist $\lambda_{1}, \cdots, \lambda_{m} \in f(X)$ such that $\lambda_{1}+V, \ldots, \lambda_{m}+$ $V$ are distinct (and hence disjoint) cosets of the subgroup $V$ and their union contains $f(X)$. Let $S_{i}=f^{-1}\left(\lambda_{i}+V\right), 1 \leqslant i \leqslant m$. Then $\left\{S_{1}, \ldots, S_{m}\right\}$ is an open partition of $X$, and $\Sigma_{i=1}^{m} \lambda_{i} \varphi_{S_{i}}-f \in V_{u}$. Consequently, as $v$ is a continuous linear form on $D(X, F)$ and as $F$ is complete, $v$ has a unique continuous extension to $C^{*}(X, F)$. 
(2) By Theorem 3, it suffices to show that for each $a \in \beta_{\mathrm{o}} X, u_{a}: f \mapsto$ $\left(\beta_{o} f\right)(a)$ is continuous for $T_{u}$. But for any $V \in V, u_{a}\left(V_{u}\right) \subseteq V$, since if $f \in$ $V_{u}$, then $f(X) \subseteq V$, whence $\left(\beta_{0} f\right)\left(\beta_{0} X\right) \subseteq V$ as $V$ is closed.

(3) For each $a \in \beta_{0} X$ we shall denote by $\mu_{a}$ the member $\mu_{u_{a}}$ of $M(X, F)$ associated to the character $u_{a}$. Then, for every $S \in S(X), \mu_{a}(S)=$ $u_{a}\left(\varphi_{S}\right)=\left(\beta_{0} \varphi_{S}\right)(a)=\varphi_{\bar{S}}(a)$, where closure refers to $\beta_{\mathrm{o}} X$; thus $\mu_{a}(S)$ is 1 or 0 according as $a \in \bar{S}$ or $a \notin \bar{S}$. Therefore $\mu_{a} \in M_{\mathrm{o}}(X, F)$. Conversely, let $\mu \in$ $M_{\mathrm{o}}(X, F)$, and let $v \in C^{*}(X, F)^{d}$ be such that $\mu_{\nu}=\mu$. We shall first show that if $S$ and $T$ are clopen sets, then $v\left(\varphi_{S} \varphi_{T}\right)=v\left(\varphi_{S}\right) v\left(\varphi_{T}\right)$. If either $\mu(S)=0$ or $\mu(T)=0$, then $\mu(S \cap T)=0$ as $\mu$ is monotone, so $v\left(\varphi_{S} \varphi_{T}\right)=\mu(S \cap T)=0=$ $\mu(S) \mu(T)=v\left(\varphi_{S}\right) \nu\left(\varphi_{T}\right)$. Suppose that $\mu(S)=\mu(T)=1$. Then $\mu(X-T)=0$ as $\mu(X-T)+\mu(T)=\mu(X)=1$, and hence $\mu(S-T)=0$ as $S-T \subseteq X-T$. Therefore $v\left(\varphi_{S} \varphi_{T}\right)=\mu(S \cap T)=1=v\left(\varphi_{S}\right) v\left(\varphi_{T}\right)$ since $\mu(S \cap T)+\mu(S-T)=$ $\mu(S)=1$. From this equality, it follows readily that $\nu$ is a character.

THEOREM 20. Let $F$ be a complete metrizable field whose open additive subgroups form a fundamental system of neighborhoods of zero, and let $X$ be an ultraregular space. (1) For each $a \in \beta_{0} X$, let $H(a)=\left\{T \subseteq X\right.$ : there is a $C_{\delta^{-}}$ set $C$ contained in either $T$ or $X-T$ such that $a \in \bar{C}\}$. Then $H(a)$ is an algebra containing all $C_{\delta}$-sets of $X$. (2) We define $\bar{\mu}_{a}: H(a) \rightarrow\{0,1\}$ by $\bar{\mu}_{a}(T)$ $=1$ if there exists $a C_{\delta}$-set $C$ contained in $T$ such that $a \in \bar{C}, \bar{\mu}_{a}(T)=0$ otherwise. Then $\bar{\mu}_{a}$ is a monotone, finitely additive function. (3) $a \in v_{0} X$ if and only if $H(a)$ is a $\sigma$-algebra, in which case $\bar{\mu}_{a}$ is countably additive.

Proof. (1) follows from Theorems 4,5 and $2^{\circ}$ of Theorem 6. (2) If $T \in H(a)$, then $\bar{\mu}_{a}(T)=1$ if and only if $\bar{\mu}_{a}(X-T)=0$ by Theorem 1. From this it follows readily that $\bar{\mu}_{a}$ is finitely additive and monotone. (3) follows from Theorem 11.

COROLlaRY. Let $B(X)$ be the o-algebra generated by the clopen subsets of $X$. For each $a \in v_{0} X$, let $\nu_{a}$ denote the restriction of $\bar{\mu}_{a}$ to $B(X)$. Then $a \mapsto v_{a}$ is a bijection from $v_{0} X$ onto the set of all monotone, countably additive functions from $B(X)$ onto $\{0,1\}$.

These theorems generalize material of [2].

5. Bornologicity of $C(X, F)$. Here we shall prove the analogue of the theorem of Nachbin [20] and Shirota [28] that $C(X, \mathrm{R})$, equipped with the topology of compact convergence, is bornological if and only if $X$ is realcompact. We assume familiarity with the terminology introduced in [30].

THEOREM 21. Let $F$ be a complete, indiscrete, nonarchimedean-valued 
field such that $|F|$ is nonmeasurable, and let $X$ be an ultraregular space. Then $C(X, F)$, equipped with the topology of compact convergence, is bornological if and only if $X$ is F-replete.

Proof. Necessity: Suppose that $X$ were not $F$-replete. By Theorem 15 there would exist $s \in v_{0} X-X$. A proof similar to the corresponding part of the proof in [20] would then establish that $h: f \mapsto\left(v_{0} f\right)(s)$ is a bounded, discontinuous linear form.

Sufficiency: Again, the proof is similar to Nachbin's proof in [20]. Let $V$ be a convex subset of $C(X, F)$ absorbing every bounded subset. A supporting set for $V$ is any compact subset $K$ of $\beta_{\mathrm{o}} X$ such that $f \in V$ whenever $f$ vanishes on $K \cap X$. The proof reduces to showing that if $z \in \beta_{0} X-X$, then there is a supporting set for $V$ to which $z$ does not belong. By hypothesis, $X=v_{0} X$, so there is a decreasing sequence $\left(C_{n}\right)_{n \geq 1}$ of clopen neighborhoods of $z$ such that $C_{1}=\beta_{0} X$ and $\bigcap_{n=1}^{\infty} C_{n} \cap X=\varnothing$. Thus $\left\{U_{n}: n \geqslant 1\right\}$ is an open partition of $X$, where $U_{n}=\left(C_{n}-C_{n+1}\right) \cap X$. Since $z \notin \beta_{0} X-C_{n}$, it suffices to show that some $\beta_{\mathrm{o}} X-C_{n}$ is a supporting set for $V$.

Suppose not. Let $|\alpha|>1$. We first show that for each $m$ there exists a continuous function not in $V$ that vanishes outside $U_{j}$ for some $j \geqslant m$. Let $k_{n}$ and $k_{n}^{\prime}$ be the characteristic functions of $C_{n} \cap X$ and $U_{n} \cap X$ respectively. As $\beta_{0} X-C_{m}$ is not a supporting set for $V$, there exists $f \notin V$ that vanishes on $X-C_{m}$. Then $\lim _{n \rightarrow \infty} \alpha^{n} f k_{n}=0$ since for any compact subset $K$ of $X$, $C_{n} \cap K=\varnothing$ and hence $\alpha^{n} f k_{n}$ vanishes on $K$ for sufficiently large $n$. Therefore $V$ absorbs $\left\{\alpha^{n} f k_{n}\right\}_{n>1}$. Let $\beta \in F$ be such that $\alpha^{n} f k_{n} \in \beta V$ for all $n \geqslant 1$. Then $\alpha^{n} \beta^{-1} f k_{n} \in V$ for all $n \geqslant 1$, so $f k_{n} \in V$ for all $n$ such that $|\alpha|^{n} \geqslant$ $|\beta|$. However, $f=f k_{m} \notin V$. Let $j$ be the greatest integer such that $f k_{j} \notin V$; then $j \geqslant m$. As $f k_{j}=f k_{j+1}+f k_{j}^{\prime}$, we conclude that $f k_{j}^{\prime} \notin V$. From this we infer that there is a strictly increasing sequence $\left(k_{j}\right)_{j>1}$ and functions $g_{j}$ such that $g_{j} \notin V$ and $g_{j}$ vanishes outside $U_{k_{j}}$. Let $h$ be defined by $h(x)=$ $\alpha^{\prime} g_{j}(x)$ if $x \in U_{k_{j}}, h(x)=0$ if $x$ belongs to any other $U_{n}$. Clearly $h$ is continuous since each $U_{n}$ is clopen. Since $B=\{f \in C(X, F):|f(x)| \leqslant|h(x)|$ for all $x \in X$ \} is bounded, there exists $\gamma \in F$ such that $B \subseteq \gamma V$. Thus as $\alpha^{j} g_{j} \in B$, we infer that $\alpha^{j} \gamma^{-1} g_{j} \in V$ and hence $g_{j} \in V$ whenever $|\alpha|^{j} \geqslant|\gamma|$. Thus we have obtained a contradiction.

\section{REFERENCES}

1. Bernhard Banaschewski, Über nulldimensionale Räıme, Math. Nachr. 13 (1955), 129-140. MR 19, 157.

2. E. Beckenstein, G. Bachman and L. Narici, Function algebras over valued fields and measures. III, IV, Atti Accad. Naz. Lincei Rend. Cl. Sci. Fis. Mat. Nat. 52 (1972), 840-845; 53 (1972), 349-358. 
3. A. Bialynicki-Birula and W. Żelazko, On the multiplicative linear functionals on the Cartesian product of algebras, Bull. Acad. Polon. Sci. Cl. III 5 (1957), 589-593. MR 19, 526.

4. Robert L. Blefko, Some classes of E-compactness, J. Austral. Math. Soc. 13 (1972), 492-500. MR 47 \#2552.

5. N. Bourbaki, Eléments de mathématique. Part. I. Livre III: Topologie générale. Chaps. 1, 2, 3ième éd., Actualités Sci. Indust., no. 1142, Hermann, Paris, 1961. MR 25 \#4480.

6. - Eléments de mathématique. Part. I. Livre III: Topologie générale. Chaps. 3, 4, 3ième éd., Actualités Sci. Indust., no. 1143, Hermann, Paris, 1960. MR 25 \#4021.

7. Eléments de mathématique. Fasc. XXX. Algèbres commutative. Chaps. 5, 6, Actualités Sci. Indust., no. 1308, Hermann, Paris, 1964. MR 33 \#2660.

8. Kim-peu Chew, A characterization of $N$-compact spaces, Proc. Amer. Math. Soc. 26 (1970), 679-682, MR 42 \#2436.

9. Shirota's theorem for N-compact spaces, Kyungpook Math. J. 12 (1972), 153-157. MR 46 \#4476.

10. R. Engelking and S. Mrówka, On E-compact spaces, Bull. Acad. Polon. Sci. Sér.

Sci. Math. Astronom. Phys. 6 (1958), 429-436. MR 20 \#3522.

11. Orrin Frink, Compactifications and semi-normal spaces, Amer. J. Math. 86 (1964), 602-607. MR 29 \#4028.

12. Leonard Gillman and Meyer Jerison, Rings of continuous functions, University Ser. in Higher Math., Van Nostrand, Princeton, N. J., 1960. MR 22 \#6994.

13. J. de Groot and J. M. Aarts, Complete regularity as a separation axiom, Canad. J. Math. 21 (1969), 96-105. MR 38 \#5160.

14. Horst Herrlich, E-kompakte Räume, Math. Z. 96 (1967), 228-255. MR 34 \#5051.

15. H. Herrlich and J. van der Slot, Properties which are closely related to compactness, Nederl. Akad. Wetensch. Proc. Ser. A $70=$ Indag. Math. 29 (1967), 524-529. MR 36 \#5898.

16. E. Hewitt, Rings of real-valued continuous functions. I, Trans. Amer. Math. Soc. 64 (1948), 45-99. MR 10, 126.

17. J. F. Kennison, Reflective functors in general topology and elsewhere, Trans. Amer. Math. Soc. 118 (1965), 303-315. MR 30 \#4812.

18. S. Mrówka, On E-compact spaces. II, Bull. Acad. Polon. Sci. Sér. Sci. Math. Astronom. Phys. 14 (1966), 597-605. MR 34 \#6712.

19. - Further results on E-compact spaces. I, Acta Math. 120 (1968), 161-185. MR 37 \#2165.

20. Leopoldo Nachbin, Topological vector spaces of continuous functions, Proc. Nat.

Acad. Sci. U.S.A. 40 (1954), 471-474. MR 16, 156.

21. Keiô Nagami, Dimension theory, Pure and Appl. Math., vol. 37, Academic Press, New York, 1970. MR 42 \#6799.

22. Peter Nyikos, Not every 0-dimensional realcompact space is $\mathbf{N}$-compact, Bull. Amer. Math. Soc. 77 (1971), 392-396. MR 43 \#8048.

23. R. S. Pierce, Rings of integer-valued continuous functions, Trans. Amer. Math. Soc. 100 (1961), 371-394. MR 24 \#A1289.

24. Paulo Ribenboim, Théorie des valuations, Les Presses de l'Université de Montréal, Montréal, 1964.

25. Prabir Roy, Failure of equivalence of dimension concepts for metric spaces, Bull.

Amer. Math. Soc. 68 (1962), 609-613. MR 25 \#5495.

26. - Nonequality of dimensions for metric spaces, Trans. Amer. Math. Soc.

134 (1968), 117-132. MR 37 \#3544.

27. Taira Shirota, $A$ class of topological spaces, Osaka Math. J. 4 (1952), 23-40. MR 14, 395.

28. - On locally convex vector spaces of continuous functions, Proc. Japan

Acad. 30 (1954), 294-298. MR 16, 275. 
29. J. van der Slot, Some properties related to compactness, Mathematical Centre .Tracts, 19, Mathematisch Centrum, Amsterdam, 1968. MR 40 \#871.

30. J. van Tiel, Espaces localement $K$-convexes. I-III, Nederl. Akad. Wetensch. Proc. Ser. A 68 = Indag. Math. 27 (1965), 249-289. MR 31 \#3841a, b; 32 \#4523.

31. Seth Warner, Characters of Cartesian products of algebras, Canad. J. Math. 11 (1959), 70-79. MR 21 \#5649.

DEPARTMENT OF MATHEMATICS, POLYTECHNIC INSTITUTE OF NEW YORK, BROOKLYN, NEW YORK 11201

DEPARTMENT OF MATHEMATICS AND SCIENCE, ST. JOHN'S UNIVERSITY, NOTRE DAME COLLEGE, STATEN ISLAND, NEW YORK 10301

DEPARTMENT OF MATHEMATICS AND COMPUTER SCIENCE, ST. JOHN'S UNIVERSITY, JAMAICA, NEW YORK 11432

DEPARTMENT OF MATHEMATICS, DUKE UNIVERSITY, DURHAM, NORTH CAROLINA 27706 